\title{
Micro-holographic methods for sub-micrometer grating fabrication in fused silica with UV femtosecond laser
}

\author{
M. Dubov ${ }^{* a}$, R. K. Nasyrov ${ }^{b}$, D.N. Nikogosyan ${ }^{c}$, \\ A. G. Poleshchuk ${ }^{\text {b }, ~ V . ~ M e z e n t s e v ~}{ }^{\text {a }}$, I. Bennion $^{\text {a }}$ \\ ${ }^{\mathrm{a}}$ Aston University, Birmingham, B4 7ET, UK \\ ${ }^{\mathrm{b}}$ Institute of Automation and Electrometry, Novosibirsk, 630090, Russia \\ ${ }^{\mathrm{c}}$ University College Cork, Cork, Ireland
}

\begin{abstract}
The optical layouts incorporating binary phase diffractive grating and a standard micro-objective were used for femtosecond microfabrication of periodical structures in fused silica. Two beams, generated in Talbot type interferometer, interfered on a surface and in the bulk of the sample. The method suggested allows better control over the transverse size of the grating pitch, and thus control the reflection strength of the waveguide or fibre grating. We present the examples of direct inscription of the sub-micrometer periodical structures using a $267 \mathrm{~nm}$ femtosecond laser radiation.
\end{abstract}

Keywords: Bragg, grating, micro-holographic, Talbot, UV, femtosecond, fused silica

\section{INTRODUCTION}

The methods of fabrication of periodic structures by femtosecond lasers, both on the surface and in the bulk of optical materials, are of great interest for a variety of photonic devices [1 - 11], such as fibre Bragg gratings (FBGs) [2-7] and photonic crystals [9-10]. Femtosecond FBGs are key elements for powerful fibre lasers [7, 12, 15]; their outstanding performance at elevated temperatures $[7,13,14]$ and overall flexibility of inscription in 3D-geometry is yet to be explored.

Few methods were suggested for femtosecond FBG fabrication by different groups. Among them, two methods are widely used: a mask method $[2,3,11,12]$, and point-by-point method $[4,6,7]$; both were demonstrated with various types of hosts and fibres. Traditionally, the mask method was proven to be the best one for CW and pulsed nano- and picoseconds UV laser inscription, but its application with femtosecond NIR lasers was found to be not simple [11]. The reason for this is that the wavelength of an NIR laser $(800 \mathrm{~nm})$ approaches the period of the mask used (typical mask period for telecom applications is $1000 \mathrm{~nm}$ ). As the broad spectrum of femtosecond lasers is spread by diffraction, a first order Bragg grating fabrication becomes difficult, if at all possible. Use of harmonics of the femtosecond laser offered a solution [2,3], however, as was demonstrated in earlier papers, the gratings created are of Type I in this case [3], which might be a disadvantage for some of the applications (for example at elevated temperatures). We believe that the adopting a double mask method [16], initially used with low coherence excimer UV lasers for $100 \mathrm{~nm}$ lithography [17], is the move in the right direction.

The point-by-point method offers more flexibility, as well as outstanding high temperature performance [7], however natural lengthening of the grating pitch leads to higher birefringence and off-resonance losses, which may (or may not) be desirable. A beam shaping technique, like placing an optical slit in front of the micro-objective [15], may help to control the transverse shape of the pitch (with respect to fibre axis), as well as its orientation. It was demonstrated recently that the point-by-point method can be implemented even for visible range applications $[5,6]$. To achieve this we suggest using a third harmonic $(267 \mathrm{~nm})$ of NIR femtosecond laser radiation and sharp focusing micro-objectives [6].

*m.dubov@aston.ac.uk; phone 4412120436 82; fax 4412120436 82; aston.ac.uk

Optical Design and Engineering III, edited by Laurent Mazuray, Rolf Wartmann, Andrew Wood, Jean-Luc Tissot, Jeffrey M. Raynor, Proc. of SPIE Vol. 7100, 71000T · (c) 2008 SPIE CCC code: 0277-786X/08/\$18 - doi: 10.1117/12.797681 
The optimum energy for circular focal spot appeared to be quite close to the threshold [6], thus there is practically no freedom left to control the transverse size of the grating pitch, as well as the induced contrast of the refractive index. A larger focal spot usually requires more laser pulse energy to initiate the inscription. The femtosecond modification using UV occurred in the presence of strong nonlinear propagation effects inside the glass, so, the transverse beam shape is even more difficult to control under these conditions, regardless of the beam shaping technique being used. It is worth to mention that the nonlinear focusing behaviour of complex 2D beams is largely unexplored, especially beyond the paraxial approximation. As a rule, one has to solve the set of nonlinear 3D-Maxwell equations numerically, which are coupled with plasma equations.

There is another "classic" method of FBG fabrication, which has not been widely used so far, namely the two beam holographic interferometry [18-20]. This method has been used to produce 1D- and 2D-structures, typically with periods of about a micrometer [8-10], and down to a few hundred of nanometers for a UV excimer or NIR femtosecond assisted surface modification [21].

In this paper we are considering three holographic optical layouts for sub-micrometer periodic structure creation, both in the bulk and on the surface. All of them are based on a combination of the diffractive optical element (DOE) and a standard micro-objective (MO). The simplest example of DOE is a binary uniform phase mask (PM). The focusing is essential for the inscription around the focal area to begin. We will continue to refer to them as micro-holographic methods.

In Table 1 we calculated half of the interference angle in a symmetrical configuration, required for inscription of the first order grating for various applications, using the formula: $\sin (\alpha)=\lambda /\left(2 \cdot \Lambda_{B}\right)$, where $\Lambda_{B}$ is the Bragg grating period of interest, which was obtained assuming $n_{\text {eff }}=1.45$. The required angle imposes demands on minimum numerical aperture (NA) of the objective. Note, that third harmonic of the titanium sapphire laser in combination with the $\times 74(\mathrm{NA}=0.65)$ micro-objective offers great flexibility for FBG inscription for applications ranging from $1550 \mathrm{~nm}$ to $650 \mathrm{~nm}$.

\begin{tabular}{|cccccc|}
\hline Application Wavelength & $1560 \mathrm{~nm}$ & 1050 & 980 & 808 & 650 \\
(Bragg period) & $(538 \mathrm{~nm})$ & $(362)$ & $(338)$ & $(279)$ & $(224)$ \\
$800 \mathrm{~nm}(\mathrm{~F})$ & 0.74 & $>1$ & $>1$ & $>1$ & $>1$ \\
$400 \mathrm{~nm}(\mathrm{SH})$ & 0.37 & 0.55 & 0.59 & 0.72 & $>1$ \\
$267 \mathrm{~nm}(\mathrm{TH})$ & 0.25 & 0.37 & 0.40 & 0.48 & 0.60 \\
\hline
\end{tabular}

Table 1. Represents single objective NA requirements to inscribe a first order FBG in a standard fibre $\left(\mathrm{n}_{\text {eff }}=1.45\right)$ depending on application wavelength (Bragg period) - shown in columns. The first, second and third rows of the table correspond to the fundamental $(800 \mathrm{~nm})$, second $(400 \mathrm{~nm})$ and third $(267)$ harmonics respectively.

This objective has a long working distance; it is fully achromatic, with a wide range of wavelengths, a high damage threshold and spherical aberration correction.

\section{OPTICAL LAYOUT}

In all examples considered in this paper we use a micro-objective in combination with DOE, which splits the incident beam into a few diffraction orders. Two beams diffracted into +1 and -1 orders are focused and combined together at the same time in focus, so the interference pattern is produced. All other diffraction orders are being blocked due to the specific design of the objective. The modification of the refractive index in the maxima of the interference pattern leads to the inscription of a periodical structure (grating). When the sample is translated perpendicularly to the interference fringes with a speed $V$, which equals to $\Lambda_{B} \cdot n \cdot f$, where $f$ is the pulse repetition rate and $n$ is an integer, a periodical structure or grating will be inscribed over the whole length of the translation. Optical schemes for first order Bragg grating fabrications using the micro-holographic method are presented in Fig.1. 

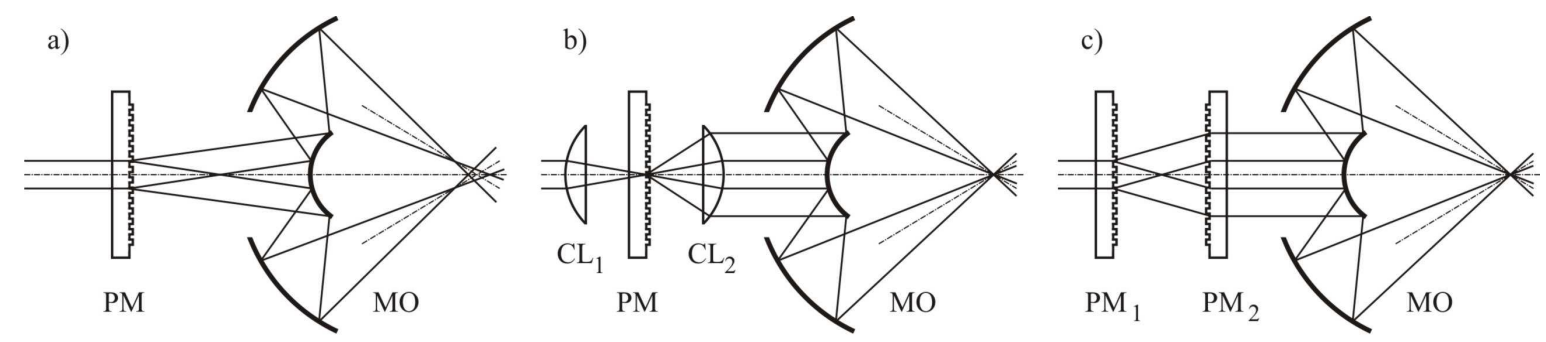

Fig. 1. Optical layouts for micro-holographic inscription: A) PM and MO pair, B) two cylindrical lenses $\left(\mathrm{CL}_{1,2}\right), \mathrm{PM}$ and $\mathrm{MO}, \mathrm{C})$ two PMs and MO.

The first version of the optical layout (Fig. 1a) consists of a pair: a phase mask and the objective. As the two beams are not parallel to the MO axis, they will be focused at different points in the focal plane. However, the beams intersect beyond the focal plane. In the area of intersection an interference pattern is produced. This scheme works well only for surface structures, but it is not possible to make an inscription in the bulk. This is caused by the fact that intensity around the focal points is higher than in the maxima of interference fringes. When the beam power is sufficient for inscription to begin, modifications (or even damaged areas, defects) are produced first in the focal plane, thus the defects start to scatter light and interference fringes will be destroyed. The scheme A is easy to align, as it has only two elements. Regardless of how short the laser pulse duration is, the pattern is created over the whole area of intersection of these two beams. This layout guarantees pulse overlapping and the generation of an interference pattern in a large volume [27, 28], even with small tilts of the mask. This geometry is suitable for inscription of micro-gratings at the surface and in the bulk of thin samples - for example, layered.

The second version of the optical layout (Fig. 1, b) includes two cylindrical lenses, the phase mask and the objective. The beam is focused by the first cylindrical lens $\mathrm{CL}_{1}$ on a phase mask PM, and then collimated after diffraction by the second cylindrical lens $\mathrm{CL}_{2}$. The position of the phase mask and the distance between $\mathrm{CL}_{1}$ and $\mathrm{CL}_{2}$ were adjusted so that the $\mathrm{PM}$ was placed in the focal plane of both lenses. Two collimated beams after $\mathrm{CL}_{2}$ fall onto the objective $\mathrm{MO}$ and, ideally, focus at the same point, where the interference pattern is produced. The application of cylindrical lenses is critical, as $\mathrm{CL}_{1}$ focuses light on the PM surface in a line with the length equal to the input beam diameter. In the case of a conventional lens, laser light is focused into a much smaller spot, thus it could damage any phase mask. With $\mathrm{CL}_{1}$ the intensity is much lower and the PM is not affected. The laser light is focused on a sample into a much smaller spot, thus the intensity in fringe maxima is sufficient to initiate the material modification, even if the sample and the PM are made from the same material. When $\mathrm{CL}_{1}$ and $\mathrm{CL}_{2}$ are different, the beam may lengthen, as well as the focal spot. The disadvantage of this scheme is in the complex adjustment, because of the small diameters of two focal spots. If the two beams are not parallel, they do not overlap, so there will be no interference pattern created. The diffraction stretches the femtosecond pulse; on top of this the direction of the wave front does not coinscide with the direction of the energy (amplitude) front. So, the stretching and the space chirp can decrease the intensity at the point of focus, thus the beam power needs to be increased. High power can damage other optical components that are used in this optical layout. Even without damage, we detected that a strong red luminescence occurred when a thick phase mask made of $8 \mathrm{~mm}$ of fused silica was used. This effect was due to the fact that the peak power of the pulse was far above the critical power for selffocusing $P_{c r}$ at this wavelength $\left(P_{c r}\right.$ scales as $\left.\lambda^{2}\right)$.

The third version of the optical layout (Fig. 1c) consists of two phase masks $\mathrm{PM}_{1}, \mathrm{PM}_{2}$ and the objective MO. After diffracting on the first and the second phase masks, the two beams become parallel. This layout, also known as Talbot interferometer [30], produces two parallel beams when the masks $\mathrm{PM}_{1}$ and $\mathrm{PM}_{2}$ are identical. In this layout two beams are being focused again at the same spot where an interference pattern is produced. Due to a small focal spot it is difficult to control the quality of alignment, similar to the previous scheme. However, it is much easier to align two masks made of plane-parallel substrates, than layout B with two cylindrical lenses. Optical layout C has a very important advantage - namely, the possibility to vary the period of the fringe pattern by changing the distance between the two masks.

Note, that all of the schemes mentioned above allow fringe period tuning by changing the wavelength of the laser light.

To illustrate the peak intensity distribution around a focal area in the case when two (broadband) Gaussian pulses arrive simultaneously at a focus in a symmetrical configuration, we integrate a standard expression of (monochromatic) field distribution over the spectrum of the laser pulse, where we have made a coordinate substitution $\{x \rightarrow x \cdot \cos (\alpha) \pm z \cdot \sin (\alpha)$; 
$z \rightarrow \pm x \cdot \sin (\alpha)+z \cdot \cos (\alpha)\}$. The results of the computer simulation of the intensity distribution are shown in Fig. 2 . Indeed, fringes have not only been formed in the focal plane $\mathrm{z}=0 \mu$, but in the vicinity of the focal region (planes marked as $\mathrm{z}=1,2,3,4 \mu$ ), therefore making the overlap with the (fibre or waveguide) core bigger.
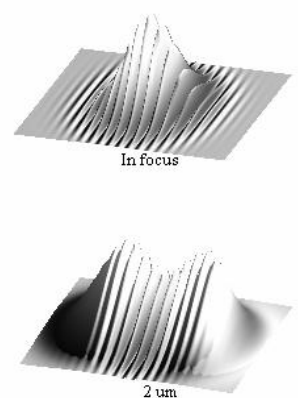
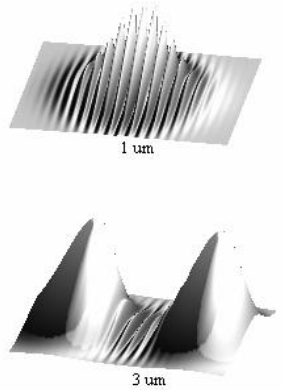
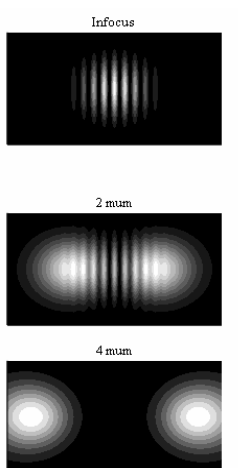
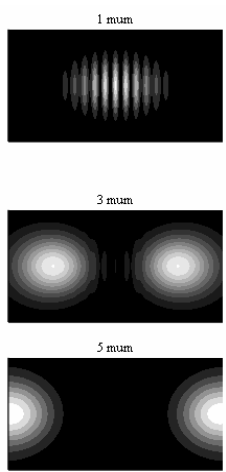

Fig. 2. a) 3D- , and b) 2D-maps of intensity distribution, plotted at a specific distance from the focal plane, as shown on the picture. Calculated fringe pattern emerged around the intersecting foci of two ultra-short Gaussian beams. The pattern was obtained by integration over the pulse spectrum, and represents time averaged fluence.

Calculations of the intensity distribution for schemes $\mathrm{B}$ and $\mathrm{C}$ require the space chirps induced by phase mask(s) to be taken into account. The analysis in both cases is straightforward [29,30], but it is beyond the scope of this paper.

We would like to mention an intrinsic achromatisation is in optical layout $\mathrm{C}$. The diffraction angle $\varphi$ on the mask is slightly bigger for the "red" part of the laser spectrum, in accordance with:

$$
\sin (\varphi)=\lambda / p
$$

where $p$ is a phase mask period and $\lambda$ is the wavelength. The beam separation after the second phase mask, positioned at the distance $L$ from the first one, would be larger for longer wavelength in accordance with:

$$
d=L \cdot \tan (\varphi)=\frac{L \cdot \lambda / p}{\sqrt{1-(\lambda / p)^{2}}}
$$

The micro-objective translates the side shift of the beam $d$, into a different incident angle $\psi$ in accordance with the equation:

$$
\tan (\psi)=A \cdot d^{2}+B \cdot d+C
$$

which we found using a ray-tracing program and manufacturer's specifications for the objective. The parameters $A, B$, and $C$ in the case of $\times 74$ objective are: $0.1558,0.1911$ and 0.075 respectively, when $d$ is in mm. Finally, the fringe period after the micro-objective would be:

$$
\Lambda_{B}=\frac{\lambda}{2 \cdot \sin (\psi)}
$$

The term "achromatisation" means that the change in Bragg period $\Lambda_{B}$ will be compensated when the laser wavelength is increased (decreased), because the angle $\psi$ also increases (decreases), in accordance with the last equation.

For the experiments described below we have not explored this effect when our masks were designed. We estimate the space-chirp to be negligible as well, due to the use of mask periods, which are much longer than the laser wavelength. The temporal pulse stretching has not been taken into account due to long pulse duration of our laser at $266 \mathrm{~nm}-$ this was estimated to be 250-350 fs (FWHM). 


\section{EXPERIMENTAL RESULTS}

We use a chirped-pulse amplification laser system (Spitfire, Spectra Physics) at $800 \mathrm{~nm}$ - a fundamental wavelength, having a spectral width of $12 \mathrm{~nm}$ (FWHM), a pulse repetition rate of $1 \mathrm{kHz}$, a pulse duration below $150 \mathrm{fs}$ (FWHM), and a pulse energy of up to $0.8 \mathrm{~mJ}$. Second and third harmonics were obtained in our home made set-up [6], by collinear and non-collinear frequency mixing for second and third harmonics respectively, in two $1 \mathrm{~mm}$ thick BBO crystals, with the conversion efficiency into the third harmonic of up to $12 \%$. We use the air-bearing stage under computer control (ABL 1000 , Aerotech) for sample translation; this has the accuracy of $50 \mathrm{~nm}$ on both $\mathrm{X}$ - and Y-axis. The reflecting objective with numerical aperture NA $=0.65$ and UV-enhanced Al-coating was used (Ealing). The set-up is essentially the same as the one described in [6]. All phase masks were specially designed and fabricated at the Institute of Automation and Electrometry (Novosibirsk, Russia), the details can be found in [22]. The periods of binary phase masks, with zero-order suppression, fabricated on fused silica substrates, used in optical layouts $\mathrm{A}, \mathrm{B}$ and $\mathrm{C}$ were $100 \mu, 50 \mu$ and $15 \mu$ respectively.

Below (Fig.3), we present a more detailed description of the layout for scheme A.

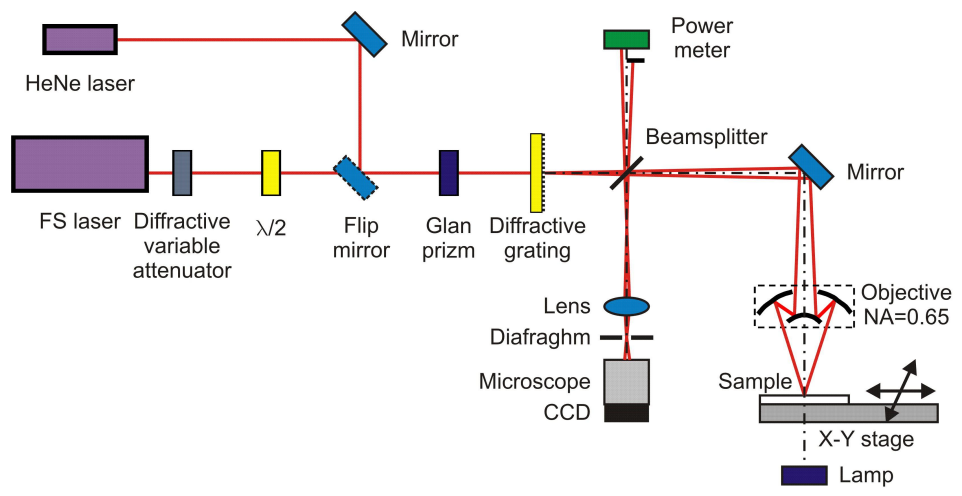

Fig. 3. Experimental set-up A (see text for details).

The distance between the objective and the $100 \mu$ phase mask was $120 \mathrm{~mm}$. The objective creates two focal points separated by $42 \mu$. The sample surface was placed at a distance of about $45 \mu$ from the focal plane. The period of the interference pattern at this distance was about $1.0 \mu$, when fundamental light was used with the wavelength of $800 \mathrm{~nm}$. We try to keep the beam power at a level that is sufficient to modify (ablate) the material of the sample (coated with Al or uncoated) only at positions where the intensity is maximal. Coarse beam attenuation was achieved by using the diffractive optical attenuator, which attenuates light up to 20 times and can withstand even more powerful lasers [24]. For fine tuning of the beam power (at $800 \mathrm{~nm}$ ) a combination of a half-wave plate and a Glan prism was used. Beam power on the sample was pre-calibrated using a beamsplitter. The beamsplitter was used for sample alignment (with an auxiliary He-Ne laser and flip mirror), as well as for on-line monitoring using a CCD camera, as the beamsplitter was installed after the phase mask. We align the orientation of the MO to maximize the area occupied by fringes, free of parasitic diffraction and obscuration by a small mirror and its three supports. In our case the interference pattern was of a specific rhombus shape (Fig.4a), where the experimentally recorded interference pattern with the period of about 1 micrometer is clearly seen. Note that the width of the interference pattern created is of the order of 20 micrometers. The on-line monitoring of the period of the interference pattern in a holographic set-up can be done as well. The same approach may be implemented for on-line monitoring of the results of a sample modification as well.
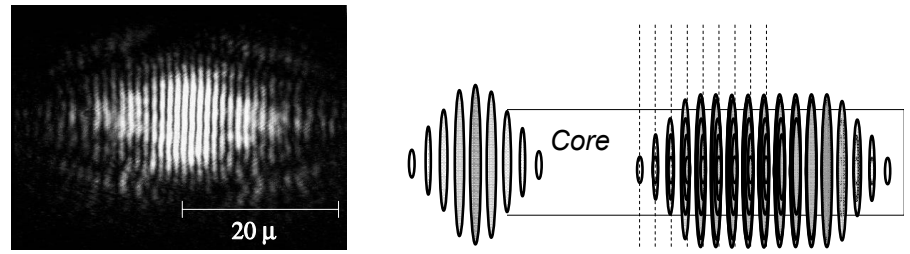

Fig. 4. The interference pattern recorded with He-Ne laser, (a), schematic of the overlap of the fringes from consecutive shots, (b). 
When we translate the sample with the speed matching the period of the interference pattern, the interference maxima from consecutive laser shots overlap with each other (Fig. 4b). Thus, for $1.07 \mu$ period of fringes, the matching translation speed should be $1.07 \mathrm{~mm} / \mathrm{sec}$, if the pulse repetition rate is $1 \mathrm{kHz}$. After the inscription, the sample was monitored with the help of the optical microscope (Axioscope 2-MOT, Zeiss).

The microscopic photos of the surface are presented in Fig. 5. Using scheme A, micro-gratings were inscribed both on bare (Fig. 5, a) and Al-covered surfaces of the fused silica slide (Fig. 5, b). The aspect ratio in the case of the surface ablation was 5:1.

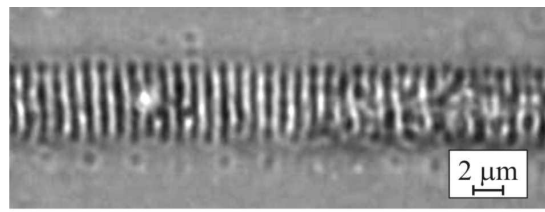

a)

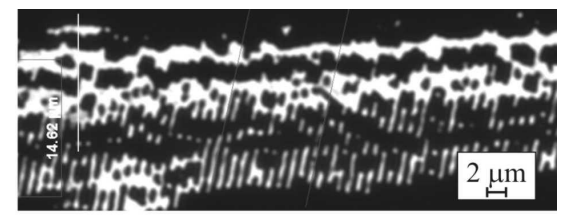

b)

Fig. 5. Micro-gratings fabricated on the surface of the glass (a); and on the surface, coated with the Al-layer, (b). Laser pulse energy was $300 \mathrm{~nJ}$ in case (a).

We believe this may allow us to obtain higher diffraction efficiency in comparison with the point-to-point method. In the case of $\mathrm{Al}$ film ablation we change the orientation of the fringes slightly. The mismatch of the speed, parasitic diffraction, and, perhaps, a re-deposition of the $\mathrm{Al}$ did not allow us to obtain "clean" ablation in this case.

Further examples demonstrate micro-gratings created using scheme B (Fig. 6) and optical layout C (Fig 7) and $267 \mathrm{~nm}$ femtosecond laser. In these experiments, the laser pulse energy on target was $160 \mathrm{~nJ}$ and $55 \mathrm{~nJ}$ respectively. The beam power and the sample translation speed were the variables to optimise. The surface modification results (obtained with $160 \mathrm{~nJ}$ ) are presented in Fig. 6 a) to f). Figure 6 a) to d), each of them is a scan with constant energy but different translation speeds: $0.4,0.5,0.6,0.7 \mathrm{~mm} / \mathrm{s}$. It turned out that best contrast was achieved at 0.6 to $0.7 \mathrm{~mm} / \mathrm{s}-$ slightly more than expected. To confirm the alignment we have done two control experiments (Fig. 6 e, f). Fig 6, e presents the result of a scan on the same sample when interference fringes are parallel to the direction of the scan (translation speed of 0.1 $\mathrm{mm} / \mathrm{s}$ ), while in Fig. $6 \mathrm{f}$ ) we have used a high scanning speed of $5 \mathrm{~mm} / \mathrm{s}$ in the direction perpendicular to the interference fringes, to resolve single shots. The five longitudinal lines in Fig. 6, e) and up to 5 lines inside the single shot spot in Fig. 6, f) confirm that interference has occurred.

This period exists within a $2.5 \mu$ focal spot area. In our previous work [6] with the focal spot of $0.4-0.5 \mu$ in diameter, the inscription (energy) threshold was measured to be about 20 to $30 \mathrm{~nJ}$. In the experiment being discussed, the laser fills half of the 5 - 6 times larger area (due to fringes), this should give us about an increase in threshold energy to $300-450$ $\mathrm{nJ}$. The estimation correlates within the factor of 2 to 4 with our experimental results.
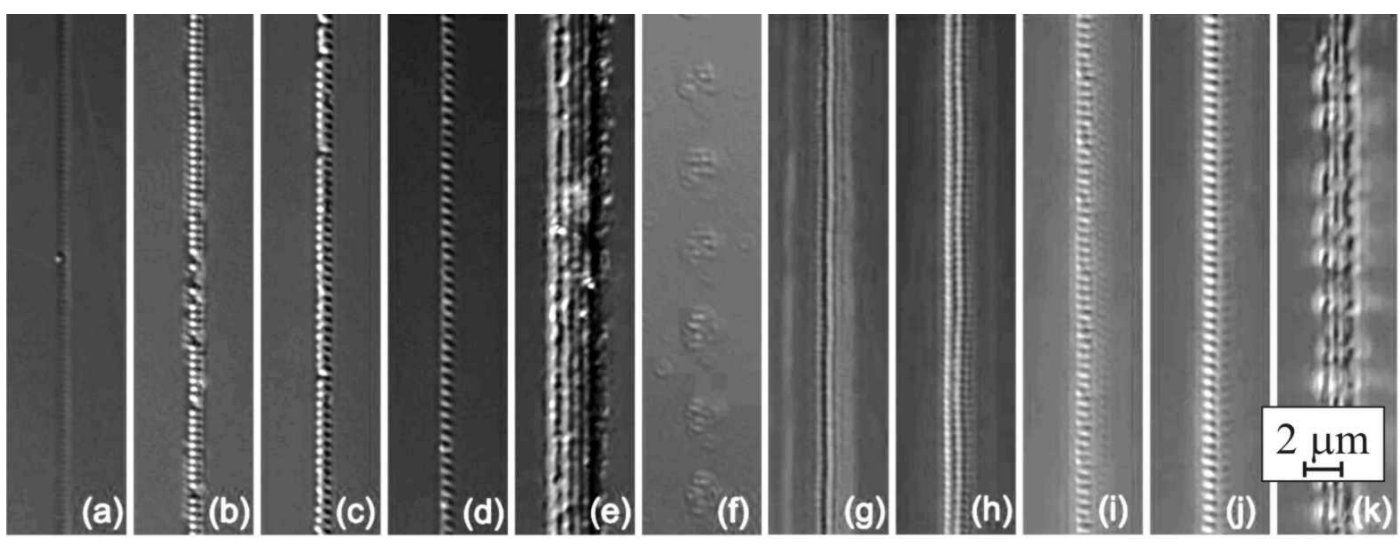

Fig. 6. Micro-structures recorded using scheme B with two cylindrical lenses of $75 \mathrm{~mm}$ focal distance. Tracks a) to f) were obtained on the surface, while g) to k) were formed in the bulk of the fused silica sample. The translation speed was: (a) 0.4 , (b) 0.5 , (c) 0.6 , (d) $0.7 \mathrm{~mm} / \mathrm{s}$, (e) translation in perpendicular direction (parallel to fringes), (f) translation with the speed of $5 \mathrm{~mm} / \mathrm{s}$ and total energy $90 \mathrm{~nJ}$. Figures g) to k) represent experimental results obtained, when focused in the bulk with the pulse energy $315 \mathrm{~nJ}$ at the depth of $75 \mu$, the speed in this case was: (g) 0.4 , (h) 0.5 , (i) 0.6 , (j) $0.7 \mathrm{~mm} / \mathrm{s}$; (k) 
represents the track obtained when the sample was translated parallel to the fringes, in this case the pulse energy was $315 \mathrm{~nJ}$.

Surprisingly, it was not possible to detect fringes on the trace of single shots when we inscribed in the bulk at the depth of $75 \mu$ (Fig. 6 g-k)). The "best contrast" was achieved again at the translation speed of 0.6 to $0.7 \mathrm{~mm} / \mathrm{sec}$, while the number of longitudinal fringes (Fig. 6, k)) was reduced to 2 or 3 . Note that at $0.4 \mathrm{~mm} / \mathrm{s}$ there was practically no periodicity seen (Fig. 6, g); at $0.5 \mathrm{~mm} / \mathrm{s}$ the structure looked like a weak grating embedded into the waveguide (Fig. 6, f). Remarkably, we achieved the best aspect ratio of about 4:1 in this case.

Perhaps, the decrease in the number of fringes was due to the presence of aberrations and nonlinear effects at higher beam power ( $315 \mathrm{~nJ}$ in the case of Fig.6, $\mathrm{k}$ this corresponds to the pulse peak power of 5 to $10 P_{c r}$ ). The aberrations may significantly distort the focal spot pattern, and lead to an increase in the threshold and, thus, the nonlinear effects.

We conclude, that both on the surface and in the bulk of the fused silica glass slide the optical layout B allowed us to create fringes within the focal spot area. The quality of the interference pattern inside the glass was poor, perhaps due to the presence of aberrations and nonlinear effects. Overall, optical layout B requires thorough alignment and elaborated procedures for beam quality control.

The experimental results of the inscription at the depth of $75 \mu$ and the laser pulse energy of $90 \mathrm{~nJ}$, in case of optical setup $\mathrm{C}$ with two phase masks, are shown in Fig. 8. The phase masks period was $15 \mu$, and the distance between $\mathrm{PM}_{1}$ and $\mathrm{PM}_{2}$ was set to be $37.6 \mathrm{~mm}$. Again, we did not managed to see a single shot pattern as clear as in the case of layout $\mathrm{B}$ (when focused on the surface, Fig 6, f).

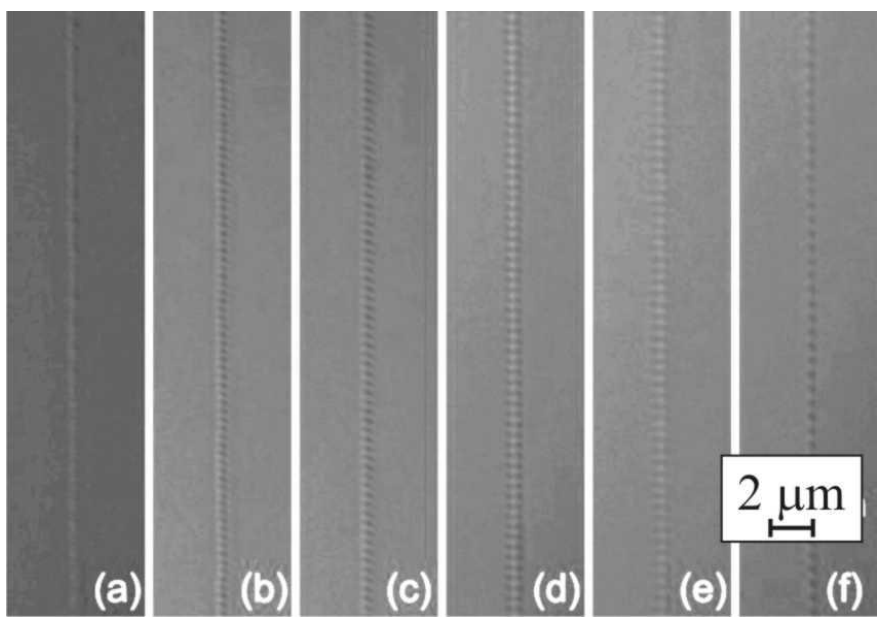

Fig. 7. The microstructures inside a fused silica sample at the depth of $75 \mu$, obtained with the translation speed of $0.4 \mathrm{~mm} / \mathrm{s}$ (a), 0.45 (b), 0.5 (c), 0.55 (d), 0.6 (e), $0.75 \mathrm{~mm} / \mathrm{s}$ (f); the pulse energy was $90 \mathrm{~nJ}$.

The maximum contrast of the periodical structure inside the fused silica sample was achieved at $0.55 \mathrm{~mm} / \mathrm{s}$. Neither the scan in the direction of interference fringes, nor the single shot patterns have revealed a presence of interference fringes. Note the quality of the periodic structure at "optimal speed" of $0.55 \mathrm{~mm} / \mathrm{s}$ (Fig.7, d). The inscription threshold was approximately two times lower compared to scheme B.

Even though scheme $\mathrm{C}$ has no cylindrical surfaces to align, it requires careful alignment as well. For example, consider an angular misalignment of the first mask over a small angle $\alpha$. The optical path difference between +1 and -1 order beams would $\mathrm{be} \approx 2 \cdot L \cdot(\mathrm{p} / \lambda)^{2} \cdot \alpha$; with the parameters used in our experiment, the angular alignment should be done with the accuracy of angular second (!) to obtain the interference. The wafer for the phase masks should be precisely plane-parallel as well. Thus, this layout has to be realized in a much more compact way. This would require a rigorous simulation of beam propagation, including temporal and space pulse chirps, as a short-period mask would introduce a significant pulse broadening [30]. The control over the temporal chirp of the TH and consideration of the nonlinear propagation effects may be required. 
The inscription in fibre samples demands our optical system for online monitoring and alignment to be improved, in spite the fact that occasionally we were able to resolve online the $\sim 0.4 \mu$ periodical structure in scheme B.

\section{CONCLUSION}

We tested three optical layouts with phase mask(s) and a micro-objective experimentally, to demonstrate the microholographic UV femtosecond inscription of sub-micrometer gratings, both on the surface and in the bulk of fused silica. These methods allow much better control of the transverse dimensions of sub-micrometer periodic structures, which is critical for applications in the visible range. In this method, the exposure in every particular point is of a cumulative nature, thus allowing an effective averaging of the laser fluence. We believe this could reduce the variation in amplitude of the induced changes of the refractive index in the material. All optical layouts considered allow on-line changes of the period, for example, by changing the wavelength of the inscription laser, or a simple mask shift or replacement.

The authors appreciate the financial support of the Engineering \& Physical Sciences Research Council in carrying out this work.

\section{REFERENCES}

[1] Canning, J., "Fibre gratings and devices for sensors and lasers, "Laser \& Photonics Review," 2(4), 275-289 (2008).

[2] Dragomir, A., Nikogosyan, D. N., Zagorulko, K. A., Kryukov, P. G., Dianov, E. M., "Inscription of fiber Bragg gratings by ultraviolet femtosecond radiation," Opt. Lett. 28 (22), 2171-2173 (2003).

[3] Slattery, S. A., Nikogosyan, D. N., Brambilla, G, "Fiber Bragg grating inscription by high-intensity femtosecond UV laser light: comparison with other existing methods of fabrication," J. Opt. Soc. Am. B 22, 354-361 (2005).

[4] Martinez, A., Dubov, M., Khrushchev, I., Bennion, I., "Direct writing of fibre Bragg gratings by femtosecond laser," Electron. Lett. 40 (19), 1170-1172 (2004).

[5] Bennion, I., Mezentsev, V., Dubov, M., Nikogosyan, D., Petrovic, J., Lai, Y., Smith, G., Zhou, K., Sugden, K., "Device Fabrication by Femtosecond Laser Inscription," BGPP-2007 Bragg Gratings Photosensitivity and Poling in Glass Waveguides (OSA Technical Digest (CD) Optical Society of America, 2007) paper BWB6 (2007).

[6] Dubov, M., Bennion, I., Nikogosyan, D.N., Bolger, P., Zayats, A.V., "Point-by-point inscription of $250 \mathrm{~nm}$ period structure in bulk fused silica by tightly focused femtosecond UV pulses," J. Opt. A Pure Appl. Opt. 10 (2), 025305 $1-6(2008)$.

[7] Jovanovic, N., Fuerbach, A., Marshall, G. D., Withford, M. J., Jackson, S. D., "Stable high-power continuous-wave Yb3+-doped silica fiber laser utilizing a point-by-point inscribed fiber Bragg grating," Opt. Lett. 32 (11), 1486-1488 (2007).

[8] Kondo, T., Juodkazis, S., Mizeikis, V., Misawa, H., Matsuo, S., "Holographic lithography of periodic two- and three-dimensional microstructures in photoresist SU-8," Opt. Express 14(17), 7943-7953 (2006).

[9] Kondo, T., Matsuo, S., Juodkazis, S. and Misawa, H., "Femtosecond laser interference technique with diffractive beam splitter for fabrication of three-dimensional photonic crystals," Appl. Phys. Lett. 79(6), 725-727 (2001).

[10] Juodkazis, S., Mizeikis, V., Matsuo, S., Ueno, K., Misawa, H., "Three-dimensional micro- and nano-structuring of materials by tightly focused laser radiation," Bull. Chem. Soc. Jpn. 81(4), 411-448 (2008).

[11] Smelser, C. W., Mihailov, S. J., Grobnic, D., Lu, P., Walker, R. B., Ding, H., Dai, X., "Multiple-beam interference patterns in optical fiber generated with ultrafast pulses and a phase mask," Opt. Lett. 29(13), 1458-1460 (2004).

[12] Wikszak, E., Thomas, J., Klingebiel, S., Ortaç, B., Limpert, J., Nolte, S., Tünnermann, A., "Linearly polarized ytterbium fiber laser based on intracore femtosecond-written fiber Bragg gratings," Opt. Lett. 32(18), 2756-2758 (2007).

[13] Martinez, A., Dubov, M., Khrushchev, I., Bennion, I., "Direct femtosecond inscription of fiber Bragg gratings," Proc. MRS 850, 137-142 (2005).

[14] Martinez, A., Khrushchev, I., Bennion, I., "Thermal properties of fiber Bragg gratings inscribed point-by-point by an infrared femtosecond laser," Electron. Lett. 41(4), 176-177 (2005).

[15] Marshall, G. D., Ams, M., Withford, M. J., "Direct laser written waveguide-Bragg gratings in bulk fused silica," Opt. Lett. 31(18), 2690-2691 (2006).

[16] Livitziis, M., and Pissadakis, S., "Bragg grating recording in low-defect optical fibers using ultraviolet femtosecond radiation and a double-phase mask interferometer," Opt. Lett. 33(13), 1449-1451 (2008). 
[17] Yen, A., Anderson, E. H., Ghanbari, R. A., Schattenburg, M. L., Smith, H. I., "Achromatic holographic configuration for 100-nm-period lithography," Appl. Opt. 31(22), 4540-4545 (1992).

[18] Kawamura, K., Sarukura, N., Hirano, M., Hosono, H., "Fabrication of surface relief gratings on transparent dielectric materials by two-beam holographic method using infrared femtosecond laser pulses," Appl. Phys. B 71(1), 119-121 (2000).

[19] Kawamura, K., Hirano, M., Kamiya, T., Hosono, H., "Femtosecond-laser-encoded distributed-feedback color center laser in lithium fluoride single crystal," J. Non-Cryst. Solids 352(23-25), 2347-2350 (2006).

[20] Kawamura, K., Ito, N., Sarukura, N., Hirano, M., Hosono, H., "New adjustment technique for time coincidence of femtosecond laser pulses using third harmonic generation in air and its application to holograph encoding system," Rev. Sci. Instrum. 73(4), 1711 (2002).

[21] Vass, C., Osvay, K., Hopp, B., "Fabrication of $150 \mathrm{~nm}$ period grating in fused silica by two-beam interferometric laser induced backside wet etching method," Opt. Express 14(18), 8354-8359 (2006).

[22] Bohme, R., Pissadakis, S., Ehrhardt, M., Ruthe, D., Zimmer, K., "Ultra-short laser processing of transparent material at the interface to liquid," J. Phys. D Appl. Phys. 39(7), 1398-1404 (2006).

[23] Poleshchuk, A. G., Churin, E. G., Koronkevich, V. P., Korolkov, V. P., Kharissov, A. A., Cherkashin, V. V., Kiryanov, V. P., Kiryanov, A. V., Kokarev, S. A., Verhoglyad, A. G., "Polar coordinate laser pattern generator for fabrication of diffractive optical elements with arbitrary structure," Appl. Opt. 38(8), 1295-1301 (1999).

[24] Poleshchuk, A. G. , Denk, D. E. , Malyshev, A. I., "High-energy diffractive variable attenuator," Proc. SPIE, 6735, 67350R (2007).

[25] Paivasaari, K., Kaakkunen, J. J. J., Kuittinen, M., Jaaskelain, T., "Enhanced optical absorptance of metals using interferometric femtosecond ablation," Opt. Express 15(21), 13838-13843 (2007).

[26] Wang, Y., Grant, J., Sharma, A., Myers, G., "Modified Talbot interferometer for fabrication of fiber-optic grating filter over a wide range of Bragg wavelength and bandwidth using a single phase mask," J. Lightwave Technol. 19(10), 1569-1573 (2001).

[27] Maznev, A. A., Crimmins, T. F., Nelson, K.A., "How to make femtosecond pulses overlap," Opt. Lett. 23(17), 13781380 (1998).

[28] Hebling, J., Kozma, I. Z., Kuhl, J., "Compact high-aperture optical setup for excitation of dynamic gratings by ultrashort light pulses," J. Opt. Soc. Am. B 17(10), 1803-1805 (2000).

[29] Ahmanov, S. A., Vislouh, V. A., Chirkin, A. S, "Optics of Femtosecond Laser Pulses," Nauka, Moscow, p. 312 (1988).

[30] Knuppertz, H., Jahns, J., Grunwald, R., “Temporal impulse response of the Talbot interferometer,” Opt. Commun. 277 (1), 67-73 (2007). 